\title{
Synthetic Biology in Cell and Organ Transplantation
}

\author{
Sean Stevens \\ Mammalian Synthetic Biology, Synthetic Genomics, Inc., La Jolla, California 92037 \\ Correspondence: sstevens@syntheticgenomics.com
}

The transplantation of cells and organs has an extensive history, with blood transfusion and skin grafts described as some of the earliest medical interventions. The speed and efficiency of the human immune system evolved to rapidly recognize and remove pathogens; the human immune system also serves as a barrier against the transplant of cells and organs from even highly related donors. Although this shows the remarkable effectiveness of the immune system, the engineering of cells and organs that will survive in a host patient over the long term remains a steep challenge. Progress in the understanding of host immune responses to donor cells and organs, combined with the rapid advancement in synthetic biology applications, allows the rational engineering of more effective solutions for transplantation.

$T^{\mathrm{h}}$ he development of synthetic biology as a discipline has greatly benefited the transplantation field. If one accepts the definition of synthetic biology as rational engineering of existing and novel biological pathways and materials, there are two broad areas of application within transplantation: the generation of organ structures from composite cells and matrices and the engineering of cellular genomes for improved survival and function in the host. The former is more relevant to organs; the latter applies to both cells and organs in transplantation.

Generation of organ and other biological structures in vitro, or tissue engineering, has been an active area of research for many years (Vacanti 2010). Application of more advanced technologies has greatly accelerated the generation of a variety of tissues and organ-like structures (Mou et al. 2015). The use of novel matrices and mechanical methods has allowed increasingly complex structures containing many different cell types (Murphy and Atala 2014). Hybrid approaches combining in vitro and in vivo organ generation are also active areas of investigation.

Modification of mammalian and other complex eukaryotic genomes has been a focus of research for decades; however, outside of mouse embryonic stem (ES) cells, most efforts have been limited to random integration of constructs in the genome using transfection or viral delivery. The application of more advanced synthetic biology techniques to mammalian genomes faces both biological and practical challenges.

First, mammalian genomes are much larger and encode substantially more genes, many of unknown function, compared with microbial genomes. Because the mammalian genome is responsible for the generation of a large variety

Editors: Daniel G. Gibson, Clyde A. Hutchison III, Hamilton O. Smith, and J. Craig Venter

Additional Perspectives on Synthetic Biology available at www.cshperspectives.org

Copyright (C) 2017 Cold Spring Harbor Laboratory Press; all rights reserved; doi: 10.1101/cshperspect.a029561

Cite this article as Cold Spring Harb Perspect Biol 2017;9:a029561 
of divergent cell types, complex regulatory circuits for gene expression are required. Therefore, predicting the effects of individual modifications on development and function of a multicellular organism is difficult.

Next, on a practical level, mammalian genomes are more difficult to modify than microbial genomes. Aside from larger genome sizes, mammalian DNA is more complex and repetitive compared with microbial genomes making synthesis and assembly more problematic. Mammalian genome engineering has accelerated as the efficiency and cost of DNA synthesis has decreased, and the size of constructs that can be produced has increased. More advanced assembly methods (Casini et al. 2015) and the use of intermediate cells such as yeast (Jakočiūnas et al. 2015) further facilitate large genome fragment construction. However, as DNA sizes increase, the isolation of intact constructs and methods for efficient delivery into cells remain limiting. The delivery of large constructs by fusion with yeast has been described, but they risk contamination from the donor cell genome ( $\mathrm{Li}$ and Blankenstein 2013). Artificial mammalian chromosomes have been constructed (Katona 2015), but their deployment in animals exhibited inaccurate mitotic division and passage to daughter cells and poor inheritance (Tomizuka et al. 2000).

Synthesis and assembly of mammalian genomic DNA is only a part of the challenge. Once novel DNA is introduced into the cell, it must be inserted at the appropriate genomic site without introducing inappropriate sequences. Engineering of mouse ES cells takes advantage of endogenous homologous recombination activity; however, other mammalian cells have required creation of site-specific double-stranded breaks in the genome and enhancement of recombination. Technologies including zinc-finger nucleases (ZFNs), transcription activator-like effector nucleases (TALENs), and CRISPR-Cas9 have allowed more efficient double-stranded DNA breaks at virtually any place in the genome (Gaj et al. 2013). CRISPR-Cas9, in particular, has created a large amount of excitement because of its ease of use compared with ZFNs and TALENs; however, in all cases, the function is limited to the creation of double-stranded DNA breaks, which, although useful, does not address the low rate of homologous recombination. Ongoing efforts seek to improve homologous recombination or circumvent these limitations with sequence-specific recombination technologies, such as recombinases and integrases (Brown et al. 2011; Turan et al. 2013).

In summary, the application of synthetic biology approaches to cell and organ generation, modification, and function has enhanced the efficiency and complexity of tissue and genome engineering, rapidly advancing the field of transplantation.

\section{FOCUS AREAS TO IMPROVE CELL AND ORGAN TRANSPLANTATION}

Transplantation is an extremely well-researched area of medicine, with many rejection mechanisms defined at the cellular and molecular level. Although this provides a broad set of pathways and targets for intervention, the scale and complexity of the modifications required have made engineering of cells and organs for transplantation extremely difficult. Most efforts have focused on either ablation of immunogenic epitopes or random integration of constitutively expressed transgenes to control rejection responses. Recent improvements in synthetic biology toolsets now make large-scale mammalian genome modification feasible.

Although the immune mechanisms involved are similar, overall rejection responses to cells and organs differ. The ability to produce and transplant relatively uniform cell populations using less-invasive technologies results in less trauma and fewer immune stimuli compared with organ transplantation involving more damaging surgeries, which activates more complex rejection responses. Therefore, the engineering required to improve survival of cells is somewhat different than for organs in transplantation. There are several broad systems to be addressed for transplantation.

\section{Reduction of Immunogenicity}

Rejection responses in the absence of pharmacologic intervention can be extremely rapid, 
with complete destruction of donor tissue in just minutes. Even syngeneic cells can be immunogenic, as shown in experiments with induced pluripotent stem cells (iPSCs) (Sackett et al. 2016). A significant number of effective pharmacologic agents have been developed for the prevention of rejection responses (Allison 2016). Although drug treatment has made kidney, liver, and heart transplants almost routine procedures, the risk of rejection is ever-present, and long-term immunosuppression increases the chance of infection and cancer. The removal of immunogenic donor epitopes, or the prevention of their recognition by the immune system, reduces the risk of rejection of the donor tissue while retaining normal immune surveillance and function.

\section{Elimination or Control of Rejection Mechanisms}

Nearly all aspects of the innate and adaptive immune responses as well as related inflammation and coagulation pathways must be addressed in transplantation. Because these systems are interdependent and synergistic, the control of key regulatory points may have broader effects upon multiple responses. For example, inflammation is linked to innate immunity and contributes to complement and coagulation activation. The tissue damage incurred releases immune stimulators and antigens, further activating innate and adaptive immunity. Improved control early in rejection limits the damage of the donor cells and tissues, and thus reduces the risk of synergistic activation of pathways that lead to rejection.

\section{Induction of Durable Tolerance}

Immunogenicity of cells and organs can be altered depending on the form of antigen presentation, the microenvironment, and regulatory molecules to induce or suppress responses to a given antigen. In addition, specialized cell populations, such as regulatory $\mathrm{T}$ cells (Tregs), can reduce or eliminate inappropriate autoimmune reactions. Several approaches take advantage of existing regulatory mechanisms to create durable tolerance to donor tissues. The potential for tolerance is particularly attractive given the undesirable side effects of long-term immunosuppression and associated risks of infection and tumorigenesis.

\section{Enhancement of Function}

The ability to carry out large-scale genome engineering allows the possibility of improving donor cell and organ functions beyond simple replacement of defective host tissues. This could be as simple as providing optimal genetic isoforms or as complex as novel engineered signaling and response pathways. Desirable characteristics such as cell activation, drug resistance, and inducibility of gene expression are all possible.

\section{CELL TRANSPLANTATION}

Transplantation of cells is more straightforward than of organs, because of lower complexity and simpler delivery. Blood transfusion has been used for hundreds of years, and bone marrow transplantation is an almost routine clinical procedure. As protocols for production and modification of therapeutic cell populations have improved, the variety of potential applications has expanded and increased the need for mechanisms to overcome rejection.

\section{Hematopoietic Cells}

Bone marrow and hematopoietic stem cell transplantation has been extremely successful creating durable functional replacement of host immune system (Pagano and Lyon 2014). Culture and modification of hematopoietic stem cells (HSCs) in vitro allows rational engineering of the immune system. A recent example is the use of genome editing to eliminate the expression of CCR5, a receptor for HIV, which controls viral infection in animal models (Holt et al. 2010). Other approaches seek to introduce broadly neutralizing antibodies to HIV, or other pathogens for which vaccination is ineffective, into HSCs for expression by the immune system (Luo et al. 2009). Because it is possible to use patient-derived HSC for modification and rein- 
S. Stevens

troduction, the risk of rejection is significantly lower than with allogeneic HSCs.

\section{$\beta$ Islet Cells}

The replacement of $\beta$ islets in diabetes has been a high-priority application of cell transplantation. Many protocols using human- and animal-derived islets have been developed for transplant, albeit with limited success because of allo- and xenogeneic rejection responses. Encapsulation of $\beta$ islets in matrices that allow the passage of smaller molecules such as insulin and glucagon, while limiting access to the immune system, have been partially effective (Ichii and Ricordi 2009). Recent data have shown that conversion of patient-derived iPSC to create syngeneic $\beta$ islets may overcome rejection responses (Quiskamp et al. 2015).

\section{Stem Cells}

Nonhematopoietic cell transplantation has been more challenging to develop in part because many terminally differentiated cell types are not easily obtained or cultured. Transdifferentiation, direct conversion of one differentiated cell type into another, sought to overcome these limitations (Wang et al. 2015); however, not all cell types are amenable to this approach, because the regulatory genes necessary for conversion are not yet known.

The identification and development of human ES cells has enhanced the creation of a number of functional cell types and organoids (Girlovanu et al. 2015). Because the use of fetal cells carries significant ethical concerns, adult stem cells and, more recently, patient-derived iPSCs have become a greater focus. Differentiation of adult stem cells and iPSCs to heart, kidney, liver, and lung tissue has been very successful; however, the production of full-size human organs has not yet been realized, because of the lack of spatial cues and appropriate morphogenesis during embryonic development, and the complexity of multicellular tissues.

Despite being syngeneic with the host, iPSCs may still be immunogenic in vivo. Generation and culture of iPSCs leads to genomic mutations and epigenetic changes that may contribute to immunogenicity (Cao et al. 2014). Immunization data show that even completely conserved proteins can elicit a response; therefore, it may not be surprising that much more complex albeit syngeneic cells may also do so.

\section{Immunotherapy}

One of the most exciting applications of cell transplantation is in the area of cancer immunotherapy. Tumor studies show a significant intratumoral infiltration of immune cells, including $\mathrm{T}$ cells specific for tumor-derived peptides. Early studies isolated, expanded, activated, and reintroduced these tumor-infiltrating lymphocytes (TILs) into patients but met with limited success (Schober and Busch 2016). An improvement to this approach targets patient-derived cells with tumor-specific T-cell receptors (TCRs) with enhanced activity to improve antitumor activities (Kessels et al. 2001). More recently, chimeric antigen receptors (CARs), consisting of high-affinity binding domains to target tumor cell surface antigens linked to signaling domains that activate T-cell antitumor functions, have shown superior activity when introduced into patient $\mathrm{T}$ cells and used to treat tumors. The stunning successes of CAR-T cell approaches have led to further variations, including improved CAR-binding specificity and signaling, enhancement of T-cell effector mechanisms as well as expansion to alternate cell types, such as natural killer (NK) cells. The successes of CAR-T cells have mainly been limited to liquid tumors, such as leukemias and lymphomas, which possess highly specific surface targets. Solid tumors present a greater challenge because of the many immunosuppressive mechanisms used and greater target heterogeneity. Although synthetic biology approaches are being applied in many aspects of cancer immunotherapy, this topic has been extensively reviewed elsewhere (Chakravarti et al. 2016).

\section{ORGAN TRANSPLANTATION}

The transplantation of organs is more complex than the transplantation of cells, and even indi- 
Synthetic Biology in Cell and Organ Transplantation

vidual organs vary in their complexity. Heart, kidney, and liver transplantations are well developed clinically with extended survival times. Organs such as the lung are more challenging because of their greater sensitivity to trauma, shorter survival time, and more frequent complications. Although robustness is important to transplantation success, availability is the more critical unmet need. As clinical technologies have steadily improved in the past decades, the availability of donor organs has only slightly increased. Therefore, the gap between need and availability continues to widen.

Synthetic biology helps to address the critical lack of organs through both tissue-engineering strategies in vitro and in vivo to create organs and the use of complex genome engineering to address the host rejection mechanisms. Because of the rapid advancements in the synthetic biology tools available and better understanding of key rejection mechanisms, these approaches could not only meet the need for organs but eventually surpass human organs in function and survival.

\section{Organ Differentiation}

The ability to recreate embryonic development in the laboratory has been a focus of biological study for many years, leading to the possible generation of organs suitable for transplantation entirely in vitro (Rustad et al. 2010). Although a number of species can develop in culture, the complexity of mammalian development has been more difficult to reproduce. Some progress has been made toward production of functional artificial wombs, but application to humans is unlikely for some time and subject to significant ethical concerns (Lupton 1997). Relatively more progress has been made in the creation of organoids and organ-like structures in vitro, with the generation of increasingly complex structures and functions (Dye et al. 2016). The use of various natural and synthetic matrices allows the assembly of larger structures, which, combined with iPSC technologies, would be compatible with patients. However, without the structural support and cues provided by embryonic morphogene- sis, the manufacture of functional adult organs entirely in vitro may be problematic.

\section{Organ Printing}

The use of 3D-printing technologies allows the deliberate printing of the final organ structure, overcoming the slower process of embryonic morphogenesis and thus creating organs tailored specifically for the patient (Patra and Young 2016). Combination of natural and synthetic scaffolds with the desired cell types in one process further improves the efficiency of production. This approach has been remarkably successful for more uniform and structural tissues such as bone and trachea (Fedorovich et al. 2010; Fishman et al. 2014). Attempts at creating more complex organ structures such as kidney or lung have proven more challenging, requiring accurate interactions between divergent cell types for function. For some organs, such as the lung, more than 40 cell types have been identified that require specific spatial positioning and cell-cell interactions to function properly (Nichols et al. 2014). As the sophistication and precision of 3D-printing techniques improves, organs of higher complexity will be possible.

\section{Decellularization-Recellularization}

An alternate approach to the generation of organs and 3D printing in vitro is to rebuild the organ structure from the genuine underlying noncellular scaffold, using cells compatible with the patient. This takes advantage of normal mechanisms of organ regeneration observed in recovery from injury, albeit at a larger scale and complexity. Starting with an existing organ, all of the cells are extracted using mild enzymatic and detergent treatments, leaving the decellularized structural elements (Song and Ott 2011). To this matrix are added new cells derived from, or compatible with, the patient, which are allowed to recellularize the structure, recreating the functional organ. Although this may allow the creation of compatible organs, there is still a requirement for the underlying matrix that needs to be addressed and presumably could be resolved with the use of porcine 
S. Stevens

organs. The advent of iPSC technology provides an avenue to produce the cells necessary of recellularization. However, as with 3D printing, more complex organs require cells of many different types, not all of which are defined. In addition, embryonic morphogenesis may be required for some facets of organ structure and function that recellularization cannot effectively reproduce.

\section{Nonhuman Blastocyst Complementation}

A limitation for producing organs in vitro is the challenge of creating the appropriate structures and functions without following normal embryonic morphogenesis. One route to overcome this limitation is the use of human stem cells to complement defective organ production in a large animal species, such as pig (Nagashima and Fukuzawa 2016). In this approach, pigs are genetically modified to prevent production of a particular organ, with human stem cells introduced at the blastocyst stage. As the embryo develops, the lineages that cannot be produced from host animal cells are replaced by the human stem cells, with a goal of creating a fully functional human organ in the animal using the proper developmental pathways (Wu and Izpisua Belmonte 2016). A potential limitation of this approach is the difficulty in producing an organ entirely derived from human cells to avoid the xenogeneic rejection response to chimeric human-animal organs. Similarly, the use of allogeneic human cells may contribute to rejection responses. Although patient-derived iPSC may be used, the time it would take to generate the functional organ may be limiting. In addition, the risk of human cells contributing to other lineages within the animal, such as neurons, creates ethical concerns that need to be addressed.

\section{Hybrid Methods}

Although the above approaches are described separately, it is possible to combine technologies to arrive at more satisfactory solutions. For example, 3D-printed or decellularized-recellularized organs could be introduced into patients at a heterotopic site for a period of time to allow full regeneration, before orthotopic transplantation. Although theoretically advantageous, particularly if the organ was generated from patient-derived cells or iPSCs, the risk incurred by multiple procedures as well as the immediacy of the need of many patients makes the practical application challenging.

\section{TRANSPLANTATION REJECTION MECHANISMS}

Outside of transplantation between identical twins, all cell and organ transplants are at risk of immune rejection. The greater the number of genetic differences, the greater the chance of a host immune response, thus allogeneic organs create less severe reactions than xenogeneic organs.

The human immune response to porcine organs starts with hyperacute rejection, because of significant preexisting titers of antiporcine antibodies inducing complement-mediated destruction of the tissue. This is followed by acute cellular and vascular rejection, also unique to xenotransplantation (Auchincloss and Sachs 1998). Chronic rejection is common to both allo- and xenotransplant, and is more readily controlled by existing pharmacological treatments (Scherer et al. 2007).

The synergies among the various aspects of the immune response lead to rapid amplification; therefore, the treatment of rejection is extremely difficult once a response begins. The goals for genome-engineering strategies are to prevent the initiation of rejection while blunting any amplification once it occurs. Synthetic biology approaches allow the modification of multiple pathways simultaneously, including altering immunogenicity, immune regulation, and tolerance with rational design and multigenic changes. Examples of the systems that must be addressed are described below.

\section{Histocompatibility}

The immune system efficiently recognizes differences in protein sequence through the interaction of TCRs with peptides in complex with 
histocompatibility proteins (HLA). In transplantation, TCR-HLA interactions play a role in both direct and indirect antigen presentation. Direct antigen presentation is host T-cell recognition of donor HLA (or SLA in the case of porcine cells) complexed with donor peptide or donor HLA alone due to the lack of negative thymic selection of host $\mathrm{T}$ cells against the foreign haplotypes. Indirect presentation is due to the uptake of donor proteins by host antigenpresenting cells and display by host HLA to host $\mathrm{T}$ cells (Gould and Auchincloss 1999). The combination of direct and indirect responses results in cytolytic and helper T-cell activation and destruction of the transplanted cells and organs. Although the use of patient-derived cells or iPSCs overcomes many of the challenges of antigen presentation, use of allo- and xenogeneic cells provides increasingly large numbers of immunogenic epitopes that generate T-cell responses.

Modification of donor HLA to match host haplotypes is a potential route to eliminating peptide-independent direct responses of alloand xenogeneic HLA. This would require rapid production of organs with patient-specific HLA haplotypes, which would not be practical for xenotransplantation but could be contemplated for in vitro organ production. However, this may have the unintended effect of enhancing the display of the immunogenic donor peptides by the modified donor cells, actually increasing host T-cell activity against the donor tissue. A complementary approach uses genome engineering to eliminate or reduce the expression of donor HLA and preventing antigen presentation (Hara et al. 2013). Although this approach reduces immunogenicity of donor cells, it increases the risk of infection and leads to "missing self" recognition by NK cells (van Bergen et al. 2009). Inhibitory receptors expressed by NK cells interact with cell surface HLA, preventing NK cells from targeting the cells. Therefore, ablation of donor HLA expression activates host NK cells causing donor cell destruction. Expression of ligands for NK inhibitory receptors, such as HLA-E or -G, will preclude NK cell activation (Crew 2007) and offset the elimination of antigen-presenting HLA from donor cells.
Eliminating antigen presentation also risks the inability to detect infection; therefore, a better option may be to edit immunogenic epitopes. Although CRISPR-Cas9 editing is useful for making small changes at multiple loci, the number of genetic changes required to target immunogenic loci in allotransplant is immense, with an even greater number for xenotransplant. The increased number of targets also increases risk of off-target mutations potentially creating unpredictable mutations.

Alteration of immunogenic sequences to match host sequences may be preferred over eliminating the expression of immunogenic loci, to avoid the loss of essential functional genes. However, changing coding sequences in simultaneous multiplexed CRISPR-Cas9 protocols is much less efficient than simply ablating expression, limiting the utility of this approach. Better prediction of immunogenicity through in silico modeling and in vitro assays could help prioritize required changes by focusing on the most highly reactive sequences. Unfortunately, the reliability of current prediction methods is insufficient for this task (Luo et al. 2015).

\section{Donor-Specific Antibodies}

The recognition of donor antigens by preexisting antibodies in host serum leads to hyperacute rejection via complement-mediated cell destruction of donor cells. One simple approach to reducing antibody-mediated mechanisms of rejection is to ablate the antigenic genes, or the pathways that create the antigens, in the case of glycan or other nonproteinaceous epitopes. Elimination of porcine xenoantigens has been successfully demonstrated with the "Gal" epitope (Gal $\alpha 1-3 \mathrm{Gal} \beta 1-(3) 4 \mathrm{GlcNAc}-\mathrm{R})$, a porcine glycan residue that is one of the most reactive xenoantigens in humans (Galili 1999). Ablation of the transferase gene responsible for adding the Gal epitope to proteins significantly reduces the recognition of porcine cells by human serum (Sandrin et al. 1994). Several additional human-reactive porcine xenoantigens have been defined, such as Neu5Gc and B4GALNT2 (Byrne et al. 2015). It is likely 


\section{S. Stevens}

that the elimination of additional xenoantigens may further reduce human antibody-mediated destruction of porcine cells; however, with each modification, there is a risk of eliminating key functions in porcine development or physiology.

\section{Complement}

Antibody-dependent cell-mediated cytotoxicity (ADCC) is due to the recognition of donor cell epitopes by natural and induced antibodies, resulting in the activation of the complement cascade and cell death (Sheen and Heeger 2015). The large diversity of antibody specificities can lead to inadvertent autoreactivity with cells in vivo; therefore, complement regulatory proteins (CRPs) are expressed that set a threshold for activation of ADCC to prevent accidental targeting (Mathern and Heeger 2015).

Human serum has significant titers of antibodies directed against porcine antigens and thus xenotransplanted cells are at high risk of antibody recognition and ADCC compared with allogeneic human cells (Schroeder et al. 2005). Furthermore, incompatibilities between porcine CRPs and their human counterparts in the complement cascade prevent appropriate regulation of complement activation leading to ADCC-derived damage to porcine tissues. The overexpression of human CRPs helps to regulate ADCC activity and has been a focus of transgenic animals for xenotransplantation (Miyagawa et al. 2010). The elimination of highly reactive antigens as described above, in combination with the expression of human CRPs, further reduces the risk of tissue destruction.

More precise methods of targeting in porcine cells allow the insertion of human CRPs under the control of the homologous porcine genes; however, normal levels of expression may be insufficient in xenotransplantation. Given the relatively greater number of antigens recognized by human antibodies, a higher level of CRP expression may be required compared with normal human tissue, and thus more dynamic methods of control of CRP expression may be required. One caution, which applies to manipulation of any porcine pathways, is that the endogenous porcine complement must be preserved to allow proper development and function of the porcine organ before removal for xenotransplantation; therefore, any engineering must take into account improvements transplantation in humans as well as maintaining proper function while in the pig.

\section{Ischemia/Reperfusion Injury}

Even before an organ is transplanted, it is subject to trauma during removal, preservation, and transport to the donor and subsequent surgery, leading to ischemia/reperfusion injury (I/ $\mathrm{RI})$. Initially, the loss of oxygenation causes interruption of metabolism and accumulation of metabolic intermediates. Later reintroduction of oxygenated blood can be even more damaging, overwhelming the antioxidant system and injuring the endothelium (Lutz et al. 2010). This induces expression of proinflammatory mediators and chemokines, followed by recruitment of neutrophils, macrophages, and other innate immune cells, rapidly causing tissue destruction and necrosis. The widespread inflammation also causes activation of the coagulation cascade and related thrombosis events.

\section{Inflammation}

In addition to I/RI mechanisms described above, the inflammatory process is reinforced by host innate immune responses. Damaged cells release innate immune activators, attracting cells to the site of the transplanted organ (Goldstein 2006). The influx of neutrophils, monocytes, macrophages, and other cells into the organ causes the release of additional proinflammatory mediators, resulting in further amplification of inflammation. Without resolution, the inflammation continues to damage tissue, continuing the cycle of activation and amplification, and inducing adaptive immune responses (Klose and Artis 2016). Unlike inflammation caused by infectious diseases, which can be resolved once the infectious agent is eliminated, the cells of the organ provide ongoing stimuli to the inflammatory response. 
Synthetic Biology in Cell and Organ Transplantation

Inflammation is a major challenge in alloand xenotransplantation regardless of source. Although transgenes have been introduced into the porcine genome to offset inflammatory responses, a greater number and variety of mechanisms need to be addressed (Ezzelarab and Cooper 2015a). Because a general reduction or elimination of inflammatory responses can create a risk of infection, a better solution is to tailor the engineering to limit the overreaction of rejection responses, while maintaining appropriate infectious disease responses. Creating genetic circuits to respond to inflammation in an inducible, self-limited manner will mimic the kinetics of a normal response, reducing pathogenic inflammation while maintaining infectious disease surveillance.

\section{Coagulation}

Activation of the coagulation cascade is a consequence of the inflammatory responses described above, leading to disseminated intravascular coagulation and thrombosis, rapid loss of platelet and clotting factors, decreased blood flow and ischemia/infarction (Schmelzle et al. 2010; Ezzelarab et al. 2015b). As with inflammation, coagulation is rapidly amplified after initiation and difficult to regulate once widespread. Similar to the complement cascade, there are a number of regulatory points in coagulation that, like CRPs, are not completely conserved between porcine and human systems and contribute to uncontrolled coagulation (Lin and Cooper 2009).

One route to improve coagulation regulation in xenotransplantation has been the overexpression of human coagulation regulators. Although there have been reports of improved coagulation regulation (Cowan et al. 2015), overexpression of the regulators can cause dysfunctions and death of modified pigs due to bleeding disorders. Improved expression constructs and integration of human regulator genes in place of the porcine genes may improve the defective phenotypes. Better regulation of expression through improved promoters and enhancers may be required. One challenge will be to implement appropriate post-xenotrans- plantation regulation while not disrupting endogenous porcine coagulation during pig development and growth.

\section{Hematopoietic Chimerism}

A particularly exciting approach to overcome transplant rejection is hematopoietic chimerism, the functional transplantation of donor hematopoietic cells, with or without secondary lymphoid tissues, to create durable immunological tolerance of the host to the donor antigens (Sykes 2007). This could significantly reduce, or even bypass, much of the rejection response and eliminate the requirement for long-term immunosuppression. Animal studies support the effectiveness of hematopoietic chimerism in allo- and xenotransplant tolerance (Vagefi et al. 2015). A key question is whether chimeric immune systems require central tolerance, peripheral tolerance, or both to be effective. In the former case, it may be essential to incorporate donor thymus tissue to allow reciprocal education of host and donor $\mathrm{T}$ cells (Barth et al. 2003). In the latter case, the ongoing production of potentially reactive $\mathrm{T}$ cells may require pharmacologic or other therapeutic treatments. A better understanding of the immunological mechanisms critical to establish and maintain tolerance will help establish protocols for clinical applications.

\section{FUTURE CHALLENGES}

The application of synthetic biology tools requires a better understanding of the genes affecting rejection to be fully effective. For example, recently published data regarding the engineering of a cell with a minimized genome shows that many of the genes required for life are of unknown function (Hutchison et al. 2016). The number of genes of unknown function will be much greater in mammalian cells and more challenging to define. More complex analytical tools are required for more precise definition of genetic mechanisms and cellular pathways. Similarly, understanding the regulation of the large number of interrelated genes must also improve to allow better prediction of 


\section{S. Stevens}

effects of genome modification upon gene networks. Significant progress in genomics, transcriptomics, proteomics, metabolomics, immunomics, and other systems biology approaches is accelerating the development of essential data sets and predictive algorithms for more rational engineering of cells.

Thus far, much of the focus of genome engineering in transplantation has been primarily devoted to ablation and humanization of existing genes. The advancement of synthetic biology tools now allows design and implementation of completely novel genes and pathways to improve cell functions. Orthogonal signaling systems, unique metabolic pathways, engineered proteins, and many other novel solutions can now be applied in mammalian cells to enhance transplant survival and function.

Although mammalian genome engineering has accelerated greatly, new and better tools are still required to make further improvements. More efficient, sequence-specific DNA recombination, more uniform and higher editing rates, and reduced off-target editing are all highly desirable.

At the cell and tissue levels, more effective in vitro methods for functional in vitro organ production could overcome the need for in vivo methods such as blastocyst complementation or porcine organ engineering, and greatly speed production. The combination of patient-specific cells and in vitro organ production could avoid the need for sophisticated genome engineering entirely.

\section{CONCLUSIONS}

Synthetic biology is opening the door to approaches for cell and organ transplantation that would have been impossible even just a few years ago. Methods to produce compatible cells and organs in vitro and in vivo combined with increasingly rapid and precise methods for genome engineering have rapidly advanced (Mou et al. 2015). Synthetic biology technologies not only allow the large-scale changes necessary to address the many issues critical donor acceptance and tolerance but could lead to organs superior in function to human organs.

\section{ACKNOWLEDGMENTS}

Thanks to Guliang Xia, Bhaskar Thyagarajan, Adam Corper and the Mammalian Synthetic Biology team at Synthetic Genomics, Inc., Martine Rothblatt, David Ayares, Carol Phelps, Marc Lorber, and Jordan Shin of United Therapeutics, Craig Venter, Oliver Fetzer, Todd Peterson, Toby Richardson, Dan Gibson, Ariel Schwartz, Leah Soriaga at Synthetic Genomics, Megan Sykes, David Sachs, Richard N. Pierson III, and Agnes Azimzadeh.

\section{REFERENCES}

Allison TL. 2016. Immunosuppressive therapy in transplantation. Nurs Clin North Am 51: 107-120.

Auchincloss H Jr, Sachs DH. 1998. Xenogeneic transplantation. Annu Rev Immunol 16: 433-470.

Barth RN, Yamamoto S, LaMattina JC, Kumagai N, Kitamura H, Vagefi PA, Awwad M, Colvin RB, Cooper DK, Sykes M, et al. 2003. Xenogeneic thymokidney and thymic tissue transplantation in a pig-to-baboon model: I. Evidence for pig-specific T-cell unresponsiveness. Transplantation. 75: 1615-1624.

Brown WR, Lee NC, Xu Z, Smith MC. 2011. Serine recombinases as tools for genome engineering. Methods 53: 372-379.

Byrne GW, McGregor CG, Breimer ME. 2015. Recent investigations into pig antigen and anti-pig antibody expression. Int J Surg 23: 223-228.

Cao J, Li X, Lu X, Zhang C, Yu H, Zhao T. 2014. Cells derived from iPSC can be immunogenic-Yes or no? Protein Cell 5: $1-3$.

Casini A, Storch M, Baldwin GS, Ellis T. 2015. Bricks and blueprints: Methods and standards for DNA assembly. Nat Rev Mol Cell Biol 16: 568-576.

Chakravarti D, Cho JH, Weinberg BH, Wong NM, Wong WW. 2016. Synthetic biology approaches in cancer immunotherapy, genetic network engineering, and genome editing. Integr Biol (Camb) 8: 504-517.

Cowan PJ, Robson SC. 2015. Progress towards overcoming coagulopathy and hemostatic dysfunction associated with xenotransplantation. Int J Surg. 23: 296-300.

Crew MD. 2007. Play it in E or G: Utilization of HLA-E and $-\mathrm{G}$ in xenotransplantation. Xenotransplantation 14: 198-207.

Dye BR, Miller AJ, Spence JR. 2016. How to grow a lung: Applying principles of developmental biology to generate lung lineages from human pluripotent stem cells. Curr Pathobiol Rep 4: 47-57.

Ezzelarab MB, Cooper DK. 2015a. Systemic inflammation in xenograft recipients (SIXR): A new paradigm in pig-to-primate xenotransplantation? Int J Surg 23: 301-305.

Ezzelarab MB, Ekser B, Azimzadeh A, Lin CC, Zhao Y, Rodriguez R, Echeverri GJ, Iwase H, Long C, Hara H, et al. 2015b. Systemic inflammation in xenograft recipients 
precedes activation of coagulation. Xenotransplantation 22: $32-47$

Fedorovich NE, Alblas J, Hennink WE, Oner FC, Dhert WJ. 2011. Organ printing: The future of bone regeneration? Trends Biotechnol 29: 601-606.

Fishman JM, Wiles K, Lowdell MW, De Coppi P, Elliott MJ, Atala A, Birchall MA. 2014. Airway tissue engineering: an update. Expert Opin Biol Ther 14: 1477-1491.

Gaj T, Gersbach CA, Barbas CF III. 2013. ZFN, TALEN, and CRISPR/Cas-based methods for genome engineering. Trends Biotechnol 31: 397-405.

Galili U. 1999. Significance of anti-Gal IgG in chronic xenograft rejection. Transplant Proc 31: 940-941.

Girlovanu M, Susman S, Soritau O, Rus-Ciuca D, Melincovici C, Constantin AM, Mihu CM. 2015. Stem cellsBiological update and cell therapy progress. Clujul Med 88: $265-271$.

Goldstein DR. 2006. Toll like receptors and acute allograft rejection. Transpl Immunol 17: 11-15.

Gould DS, Auchincloss H Jr. 1999. Direct and indirect recognition: The role of MHC antigens in graft rejection. Immunol Today 20: 77-82.

Hara H, Witt W, Crossley T, Long C, Isse K, Fan L, Phelps CJ, Ayares D, Cooper DK, Dai Y, Starzl TE. 2013. Human dominant-negative class II transactivator transgenic pigs-Effect on the human anti-pig T-cell immune response and immune status. Immunology 140: 39-46.

Holt N, Wang J, Kim K, Friedman G, Wang X, Taupin V, Crooks GM, Kohn DB, Gregory PD, Holmes MC, et al. 2010. Human hematopoietic stem/progenitor cells modified by zinc-finger nucleases targeted to CCR 5 control HIV-1 in vivo. Nat Biotechnol 28: 839-847.

Hutchison CA III, Chuang RY, Noskov VN, Assad-Garcia N, Deerinck TJ, Ellisman MH, Gill J, Kannan K, Karas BJ, Ma L, et al. 2016. Design and synthesis of a minimal bacterial genome. Science 351: aad6253.

Ichii H, Ricordi C. 2009. Current status of islet cell transplantation. J Hepatobiliary Pancreat Surg 16: 101-112.

Jakočiūnas T, Rajkumar AS, Zhang J, Arsovska D, Rodriguez A, Jendresen CB, Skjødt ML, Nielsen AT, Borodina I, Jensen MK, et al. 2015. CasEMBLR: Cas9-facilitated multiloci genomic integration of in vivo assembled DNA parts in Saccharomyces cerevisiae. ACS Synth Biol 4: $1226-1234$.

Katona RL. 2015. De novo formed satellite DNA-based mammalian artificial chromosomes and their possible applications. Chromosome Res 23: 143-157.

Kessels HW, Wolkers MC, van den Boom MD, van der Valk MA, Schumacher TN. 2001. Immunotherapy through TCR gene transfer. Nat Immunol 2: 957-961.

Klose CS, Artis D. 2016. Innate lymphoid cells as regulators of immunity, inflammation and tissue homeostasis. Nat Immunol 17: 765-774.

Li L, Blankenstein T. 2013. Generation of transgenic mice with megabase-sized human yeast artificial chromosomes by yeast spheroplast-embryonic stem cell fusion. Nat Protocol 8: 1567-1582.

Lin CC, Cooper DK, Dorling A. 2009. Coagulation dysregulation as a barrier to xenotransplantation in the primate. Transpl Immunol 21: 75-80.
Luo H, Ye H, Ng HW, Shi L, Tong W, Mendrick DL, Hong H 2015. Machine learning methods for predicting HLApeptide binding activity. Bioinform Biol Insights 9: 21-29.

Luo XM, Maarschalk E, O'Connell RM, Wang P, Yang L, Baltimore D. 2009. Engineering human hematopoietic stem/progenitor cells to produce a broadly neutralizing anti-HIV antibody after in vitro maturation to human B lymphocytes. Blood 113: 1422-1431.

Lupton ML. 1997. Artificial wombs: Medical miracle, legal nightmare. Med Law 16: 621-633.

Lutz J, Thürmel K, Heemann U. 2010. Anti-inflammatory treatment strategies for ischemia/reperfusion injury in transplantation. J Inflamm (Lond) 7: 27.

Mathern DR, Heeger PS. 2015. Molecules great and small: The complement system. Clin J Am Soc Nephrol 10: $1636-1650$.

Miyagawa S, Yamamoto A, Matsunami K, Wang D, Takama Y, Ueno T, Okabe M, Nagashima H, Fukuzawa M. 2010. Complement regulation in the GalT KO era. Xenotransplantation 17: 11-25.

Mou L, Chen F, Dai Y, Cai Z, Cooper DK. 2015. Potential alternative approaches to xenotransplantation. Int J Surg 23: $322-326$.

Murphy SV, Atala A. 3D bioprinting of tissues organs. Nat Biotech 32: 773-785.

Pagano L, Lyon S. 2014. Celebrating 40 years of progress in bone marrow transplantation: A report from the 40th Annual Meeting of the European Society for Blood and Marrow Transplantation. Future Microbiol 9: 1117-1121.

Nagashima H, Matsunari H. 2016. Growing human organs in pigs-A dream or reality? Theriogenology. 86: 422426.

Nichols JE, Niles JA, Vega SP, Argueta LB, Eastaway A, Cortiella J. 2014. Modeling the lung: Design and development of tissue engineered macro- and micro-physiologic lung models for research use. Exp Biol Med (Maywood) 239: 1135-1169.

Patra S, Young V. 2016. A review of 3D printing techniques and the future in biofabrication of bioprinted tissue. Cell Biochem Biophys 74: 93-98.

Quiskamp N, Bruin JE, Kieffer TJ. 2015. Differentiation of human pluripotent stem cells into $\beta$-cells: Potential and challenges. Best Pract Res Clin Endocrinol Metab 29: 833-847.

Rustad KC, Sorkin M, Levi B, Longaker MT, Gurtner GC. 2010. Strategies for organ level tissue engineering. Organogenesis 6: 151-157.

Sackett SD, Brown ME, Tremmel DM, Ellis T, Burlingham WJ, Odorico JS. 2016 Modulation of human allogeneic and syngeneic pluripotent stem cells and immunological implications for transplantation. Transplant Rev (Orlando) 30: 61-70.

Sandrin MS, McKenzie IF. 1994. Gal $\alpha(1,3)$ Gal, the major xenoantigen(s) recognised in pigs by human natural antibodies. Immunol Rev 141: 169-190.

Scherer MN, Banas B, Mantouvalou K, Schnitzbauer A, Obed A, Krämer BK, Schlitt HJ. 2007. Current concepts and perspectives of immunosuppression in organ transplantation. Langenbecks Arch Surg 392: 511-523. 


\section{S. Stevens}

Schmelzle M, Schulte Esch J II, Robson SC. 2010. Coagulation, platelet activation and thrombosis in xenotransplantation. Curr Opin Organ Transplant 15: 212-218.

Schober K, Busch DH. 2016. TIL 2.0: More effective and predictive T-cell products by enrichment for defined antigen specificities. Eur J Immunol 46: 1335-1339.

Schroeder C, Allan JS, Nguyen BN, Wu G, Zhang T, Azimzadeh AM, Madsen JC, Schuurman HJ, Sachs DH, Pierson RN III. Hyperacute rejection is attenuated in GalT knockout swine lungs perfused ex vivo with human blood. Transplant Proc 37: 512-513.

Sheen JH, Heeger PS. 2015. Effects of complement activation on allograft injury. Curr Opin Organ Transplant 20: $468-475$.

Song JJ, Ott HC. 2011. Organ engineering based on decellularized matrix scaffolds. Trends Mol Med 17: 424-432.

Sykes M. 2007. Immune tolerance: mechanisms and application in clinical transplantation. J Intern Med 262: $288-310$.

Tomizuka K, Shinohara T, Yoshida H, Uejima H, Ohguma A, Tanaka S, Sato K, Oshimura M, Ishida I. 2000. Double trans-chromosomic mice: Maintenance of two individual human chromosome fragments containing Ig heavy and $\kappa$ loci and expression of fully human antibodies. Proc Natl Acad Sci 97: 722-727.

Turan S, Zehe C, Kuehle J, Qiao J, Bode J. 2013. Recombinase-mediated cassette exchange (RMCE) - A rapidlyexpanding toolbox for targeted genomic modifications. Gene 515: 1-27.

Vacanti J. 2010. Tissue engineering and regenerative medicine: From first principles to state of the art. J Pediatr Surg 45: 291-294.

Vagefi PA, Shah JA, Sachs DH. 2015. Progress towards inducing tolerance of pig-to-primate xenografts. Int J Surg 23: 291-295.

van Bergen J, Thompson A, Retière C, Trowsdale J, Koning F. 2009. Cutting edge: Killer Ig-like receptors mediate "missing self" recognition in vivo. J Immunol 182: 2569-2572.

Wang H, Li X, Gao S, Sun X, Fang H. 2015. Transdifferentiation via transcription factors or microRNAs: Current status and perspective. Differentiation 90: 69-76.

Wu J, Izpisua Belmonte JC. 2016. Interspecies chimeric complementation for the generation of functional human tissues and organs in large animal hosts. Transgenic Res 25: 375-384. 


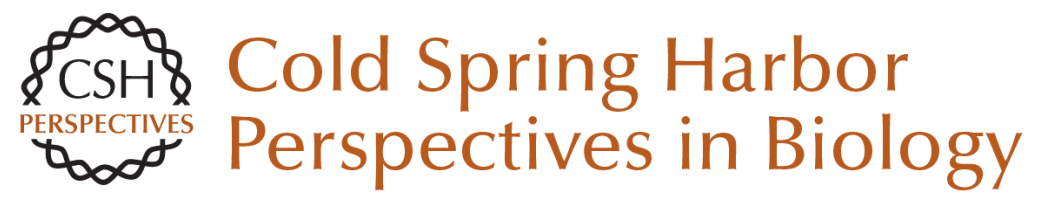

\section{Synthetic Biology in Cell and Organ Transplantation}

Sean Stevens

Cold Spring Harb Perspect Biol 2017; doi: 10.1101/cshperspect.a029561 originally published online December 21, 2016

\section{Subject Collection Synthetic Biology}

Minimal Cells--Real and Imagined John I. Glass, Chuck Merryman, Kim S. Wise, et al.

Synthetic Botany

Christian R. Boehm, Bernardo Pollak, Nuri Purswani, et al.

Synthetic Biology in Cell and Organ

Transplantation Sean Stevens

Genome-Editing Technologies: Principles and Applications

Thomas Gaj, Shannon J. Sirk, Sai-lan Shui, et al.

Alternative Watson-Crick Synthetic Genetic

Systems

Steven A. Benner, Nilesh B. Karalkar, Shuichi Hoshika, et al.

Phage Therapy in the Era of Synthetic Biology

E. Magda Barbu, Kyle C. Cady and Bolyn Hubby

Synthetic Morphogenesis

Brian P. Teague, Patrick Guye and Ron Weiss

Engineering Gene Circuits for Mammalian CellBased Applications

Simon Ausländer and Martin Fussenegger
Synthetic DNA Synthesis and Assembly: Putting the Synthetic in Synthetic Biology

Randall A. Hughes and Andrew D. Ellington

Design Automation in Synthetic Biology

Evan Appleton, Curtis Madsen, Nicholas Roehner, et al.

Cell-Free Synthetic Biology: Engineering Beyond the Cell

Jessica G. Perez, Jessica C. Stark and Michael C. Jewett

The Need for Integrated Approaches in Metabolic

Engineering

Anna Lechner, Elizabeth Brunk and Jay D. Keasling

Synthetic Biology of Natural Products

Rainer Breitling and Eriko Takano

At the Interface of Chemical and Biological

Synthesis: An Expanded Genetic Code Han Xiao and Peter G. Schultz

Building Spatial Synthetic Biology with

Compartments, Scaffolds, and Communities Jessica K. Polka, Stephanie G. Hays and Pamela A. Silver

For additional articles in this collection, see http://cshperspectives.cshlp.org/cgi/collection/

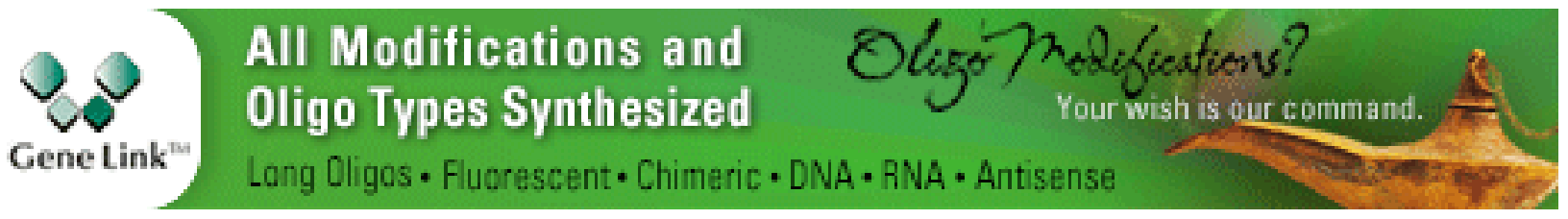

